\title{
Machine Detector Interface for the e+e- Future Circular Collider
}

\section{Manuela Boscolo*†}

Istituto Nazionale Fisica Nucleare Frascati (IT)

E-mail: manuela.boscolo@lnf.infn.it

The design of the interaction region (IR) of the positron-electron future circular collider must comply with various important constraints, imposed by high beam currents, short bunches, high beam energy, high luminosity need for polarization, and crossing scheme. The innovative IR layout of the FCC-ee is based on the crab-waist collision scheme and it will be compatible for all beam energies foreseen, from 175 to $45.6 \mathrm{GeV}$. The optics scales with the energy. We show how the latest layout for the interaction region fulfils all requirements and physical constraints, as confirmed by numerical simulations performed on critical topics such as synchrotron radiation, trapped modes, collective effects, etc. The present magnet layout including solenoid compensation scheme is discussed, together with the design of the luminosity calorimeter and the designs for the first IR quadrupole design. A refined Geant4 model for the MDI is described, which allows studying the impact of luminosity and beam backgrounds on the detector occupancy.

EPS-HEP 2017, European Physical Society conference on High Energy Physics 5-12 July 2017

Venice, Italy

*Speaker.

${ }^{\dagger}$ A footnote may follow. 
luminosity target values slightly lower now (they include $01 \%$ margin, and top is also lower due to the higher energy)

is about $10 \mathrm{~m}$ [I think it has increase after the latest match in geometry, and moving the collider rings towards the outside; reported by Katsunobu]

for photons emitted $260 \mathrm{~m}$ from the IP

\section{Interaction region layout and beam optics design}

FCC-ee is a $100 \mathrm{~km} \mathrm{e}^{+} \mathrm{e}^{-}$collider, being designed within the Future Circular Collider Study for precision studies and rare decay observations in the range of 90 to $365 \mathrm{GeV}$ centre-of-mass energy [1]. This energy range will allow precision measurements of the $\mathrm{Z}$ and $\mathrm{W}$ bosons with unique accuracy as well as explore the high energy frontier up to the Higgs boson and top quark. The high luminosities necessary to reach these physics goals require an innovative scheme of the interaction region as well as aggressive parameters. Table 1 reports beam parameters relevant for the machine detector interface studies. Given the ring circumference of $100 \mathrm{~km}$ and the limit on the synchrotron radiation power to $50 \mathrm{MW}$ per beam, the maximum design beam energy is the ttbar centre-of-mass energy. The design luminosity is higher at the $\mathrm{Z}$ energy (larger than $2 \cdot 10^{36} \mathrm{~cm}^{-2} \mathrm{~s}^{-1}$ ) and larger than $1.3 \cdot 10^{34} \mathrm{~cm}^{-2} \mathrm{~s}^{-1}$ at the top.

The interaction region is one of the key issues of a collider and the constraints from the accelerator design need to balance the detector requirements. The final goal of the design is to maximize the luminosity to background ratio. The crab-waist collision scheme [3] has been chosen for the interaction region design. The full horizontal crossing angle is $30 \mathrm{mrad}$ and local chromaticity correction is considered for the vertical plane. The design of the beam optics is described in Ref. [4] and Ref. [5] . The geometry of the FCC main tunnel is defined by the FCC hadron collider. The displacement of the FCC-ee IP with respect to the FCC-hh central trajectory is about $10 \mathrm{~m}$ and the maximum displacement between the two machines is about $12 \mathrm{~m}$ which is located about $400 \mathrm{~m}$ from the IP. The main guideline for the IR optics has been to keep the synchrotron radiation backgrounds sustainable by the detector, where this limit has been determined by the experience from the LEP2 accelerator. There the highest local critical energy was $72 \mathrm{keV}$ for photons emitted 260 $\mathrm{m}$ from the IP [6]. Consequently, main guideline in the IR design has been to keep critical energies from bending magnets below $100 \mathrm{keV}$ up to $500 \mathrm{~m}$ from the IP for the incoming beam and first dipoles located at least $100 \mathrm{~m}$ from the IP. An asymmetric optics has been designed to fulfil the requirement on the critical energy of the synchrotron radiation photons in the presence of a large crossing angle. The asymmetry allows the beam to come from the inner ring to the IP, then it is bent strongly after the IP to merge back close to the opposing ring. An additional requirement on the optics design that comes from considerations of synchrotron radiation is to keep all critical energies around the ring below $1 \mathrm{MeV}$ in order to minimize neutron production. The expected luminosity lifetime is limited by radiative Bhabha scattering and beamstrahlung to less than one hour. Thus, to increase the average luminosity, the efficient operation requires continuous top-up injection at full collision energy. In addition, the collider ring must have enough dynamical aperture to store the colliding beam, whose energy spread is drastically increased by beamstrahlung. The local chromaticity correction section is needed to reach the energy acceptance requirement of $2.5 \%$ at the ttbar energy. To mitigate the coherent beam-beam instabilities at the $\mathrm{Z}[7,8,9]$, the 
$\beta_{x}^{*}$ has been reduced from 50 to the present value $15 \mathrm{~cm}$ (see Table 1). This optics modification is obtained dividing QC1 in three independent sections and changing polarity to the section closest to IP. In this way the IR final focus quadrupole has the flexibility needed to obtain the required parameters over the entire beam energy range, maximizing the performance.

IR optics scales with the energy, allowing the IR layout to be common for all energies. Two interaction regions are foreseen in the FCC-ee collider. FCC-ee is designed with two separated beam pipes that are merged only in the IR to cross at an angle in the IP. The distance between the IP and the entrance of the first quadrupole $\mathrm{L}^{*}$ is $2.2 \mathrm{~m}$. The final focus scheme is symmetrical, optics is asymmetric only as far as incoming bend radiation is concerned. An expanded horizontal view of the FCC-ee IR layout $\pm 2.5 \mathrm{~m}$ from IP is shown in Figure 1 with all the main components [10]. The two beam pipes are merged in the central pipe as shown in the plot. The central beam pipe is $12.5 \mathrm{~cm}$ long with a radius of $15 \mathrm{~mm}$. The luminosity monitor is magenta in the plot and is placed longitudinally from 1 to $1.2 \mathrm{~m}$ from the IP. The first final focus quadrupole QC1 is shown in yellow. Synchrotron radiation mask tips are placed just in front of QC1 to intercept SR scattered particles. To reduce the multiple scattering effects in the luminosity monitor the vacuum chamber from $\pm 0.9 \mathrm{~m}$ from the IP will be in Beryllium followed by a copper vacuum chamber with proper coating to prevent electron cloud build-up. Outside this copper pipe, between the luminosity monitor window and QC1, a high-Z material shielding is needed to protect the experiment and $1 \mathrm{~cm}$ of Tantalum is sufficient as confirmed by full GEANT 4 simulation of the subdetectors (left plot of Figure 5 in section 4). In Figure 1 this shielding is shown in green. The full physical aperture of the beam pipe is $30 \mathrm{~mm}$ up to QC1. The physical aperture at QC2 is larger, $40 \mathrm{~mm}$ diameter, and then it enlarges again to $60 \mathrm{~mm}$. The space defined beyond the angle of $\pm 100 \mathrm{mrad}$ is needed by the detector, the region below this value is for accelerator use.

The interaction region layout in Figure 1 shows also the longitudinal position of the HOM ab-

\begin{tabular}{|c|c|c|c|c|}
\hline & $\mathrm{Z}$ & $\mathrm{W}$ & $\mathrm{H}$ & ttbar \\
\hline circumference $(\mathrm{km})$ & \multicolumn{4}{|c|}{97.75} \\
\hline crossing angle at IP (mrad) & \multicolumn{4}{|c|}{30} \\
\hline$L^{*}(\mathrm{~m})$ & \multicolumn{4}{|c|}{2.2} \\
\hline Total SR power (MW) & \multicolumn{4}{|c|}{100} \\
\hline Beam energy $(\mathrm{GeV})$ & 45.6 & 80 & 120 & 182.5 \\
\hline Luminosity/IP $\left(10^{34} \mathrm{~cm}^{-2} \mathrm{~s}^{-1}\right)$ & $>200$ & $>30$ & $>7$ & $>1.3$ \\
\hline$\beta_{x}^{*}(\mathrm{~m})$ & 0.15 & 0.2 & 0.3 & 1 \\
\hline$\beta_{y}^{*}(\mathrm{~mm})$ & 0.8 & 1 & 1 & 2 \\
\hline$\varepsilon_{x}(\mathrm{~nm})$ & 0.27 & 0.28 & 0.63 & 1.45 \\
\hline beam current (mA) & 1390 & 147 & 29 & 5.4 \\
\hline particles/ bunch $\left(10^{11}\right)$ & 1.7 & 1.5 & 1.5 & 2.8 \\
\hline bunch number /beam (\#) & 16640 & 2000 & 393 & 39 \\
\hline SR energy loss/turn (GeV) & 0.036 & 0.34 & 1.72 & 9.21 \\
\hline bunch length by SR /BS (mm) & $3.5 / 12.1$ & $3.3 / 7.65$ & $3.15 / 4.9$ & $2.5 / 3.3$ \\
\hline energy acceptance $(\%)$ & 1.3 & 1.3 & 1.5 & 2.5 \\
\hline
\end{tabular}

Table 1: FCC-ee beam parameters most related to IR design. 
sorbers that will lie in the vertical plane to absorb electro-magnetic fields that may be propagated or trapped in the IR. A professional CAD design of the complicated IR geometry (see Figure 2) has been performed for more realistic numerical studies with CST and HFSS and the HOM absorbers design is in progress, following the PEP-II experience [11]. The beam pipe will be warm and water cooling is needed to dissipate the power deposited on the HOM absorbers due to absorption of electro-magnetic fields.

\section{Synchrotron radiation studies}

Two independent approaches have been used to evaluate the synchrotron radiation from bends as well as from final focus quadrupoles, to design the interaction region with beam pipe dimensions, to place masks and shieldings at proper locations. MDISim [12] is used here to evaluate synchrotron radiation from near and far bends, while modified version of SYNC_BKG is used mainly to evaluate synchrotron radiation from final focus quadrupoles and to design the interaction region with masks and shieldings. In this second method beam macro-particles are traced through sliced magnets, while MDISim combines the standard tools MAD-X, ROOT and GEANT4. See Ref. [13] for a complete description of the two methods and studies.

Figure 3 shows an example of the MDISim simulation results: on the left plot is the 3D MDISim display of a Gaussian positron beam at $175 \mathrm{GeV}$ where five thousand particles are tracked from $510 \mathrm{~m}$ to the IP in Geant 4 with the standard electro-magnetic processes; on the right plot is the resulting distribution of the generated photons.

\section{Solenoid compensation scheme}

The large crossing angle with the request of a vertical emittance in the pm scale requires a solenoid compensation scheme to cancel the effect on the beam of the $2 \mathrm{~T}$ detector solenoidal

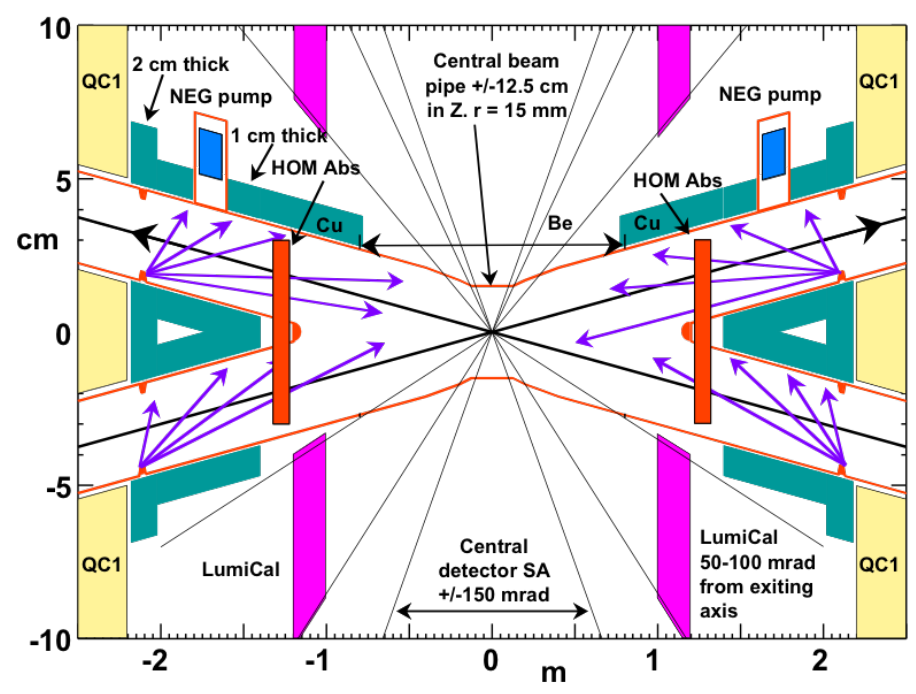

Figure 1: $\mathrm{x}-\mathrm{z}$ view of the FCC-ee IR layout for $\pm 2.5 \mathrm{~m}$ from IP. 


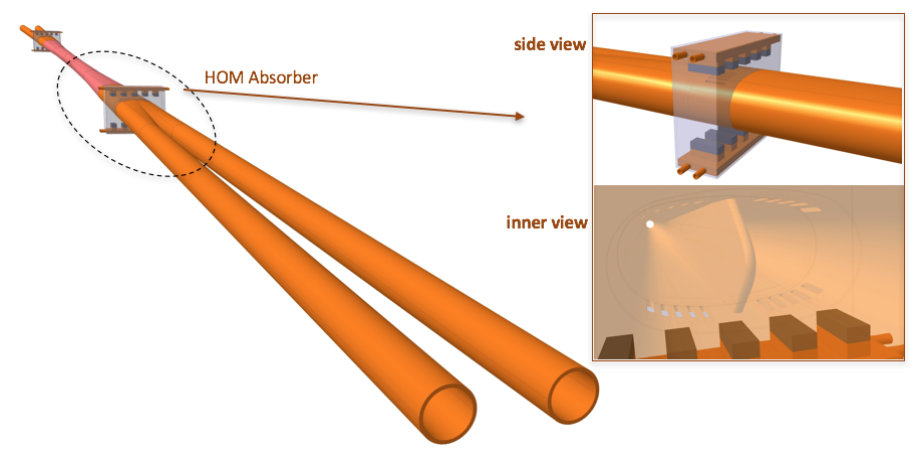

Figure 2: CAD modelling of the interaction region for the HOM design.

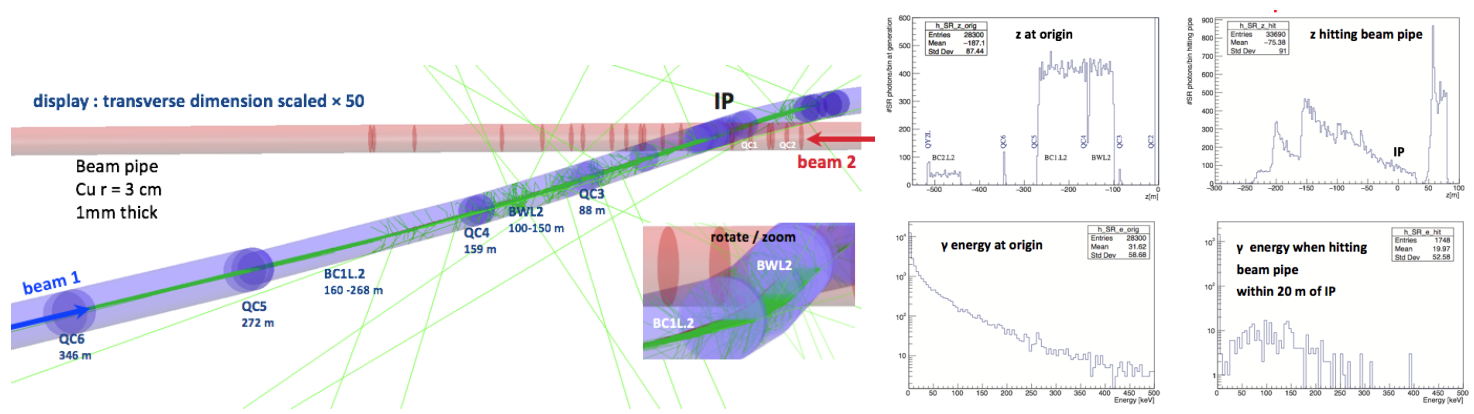

Figure 3: MDISim simulation. 3d display (left); distribution of the generated IR photons (right).

field. A compensating solenoid is placed in front of the first final focus quadrupole (QC1) to reduce the horizontal emittance blow-up and a screening solenoid shields the detector field inside the quadrupoles. This scheme is shown in Figure 4 [14]. The compensating solenoid is placed longitudinally at $1.25 \mathrm{~m}$ from the IP, just after the luminosity monitor. The overall horizontal emittance blow-up for two interaction points due to the solenoidal field is about $\approx 0.3 \mathrm{pm}$ at the $\mathrm{Z}$ energy.

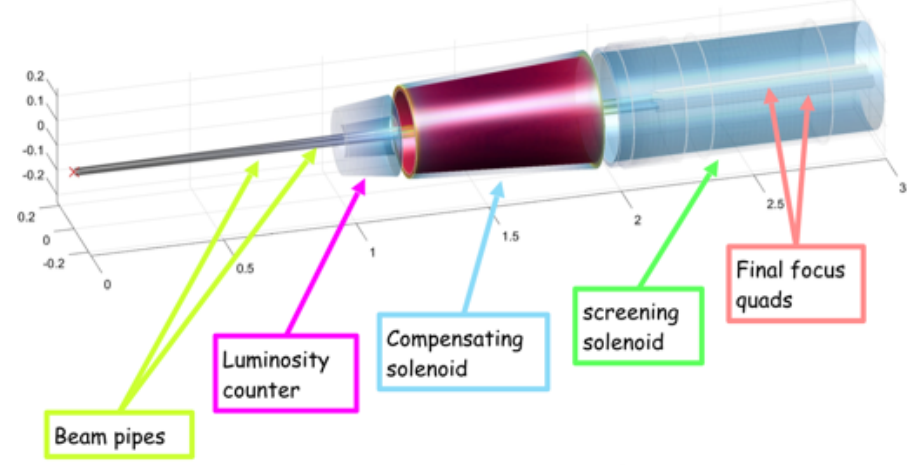

Figure 4: $3 \mathrm{~d}$ plot with the compensating and screening solenoid. 


\section{Detector and IR modeling}

Luminosity and beam induced background sources into the detector are being considered for the different running energies together with masks, shieldings and collimators. Full simulation studies of the effects of luminosity backgrounds like $\gamma \gamma \rightarrow$ hadrons and pair production in each subdetector have been performed, estimating hit density, occupancy, deposited energy [15]. Special care is reserved to the vertex detector, tracker and to the luminosity monitor. The model consists of the FCC-ee interaction region elements, around of which, a modified CLIC detector [16] has been placed. The focus of these studies is on the top energy case, which represents the worst case scenario for most of the considered backgrounds. The dominant source of primary background in the detector is synchrotron radiation: tantalum shielding is necessary to limit its impact. Left plot of Figure 5 shows the hits per bunch crossing due to synchrotron radiation with and without $1 \mathrm{~cm}$ of Ta shielding. The combined effect of synchrotron radiation and $\mathrm{e}^{+} \mathrm{e}^{-}$pairs background is shown in the right plot of Figure 5, where the maximum hit density on the hottest area of each subdetector per bunch crossing is shown.
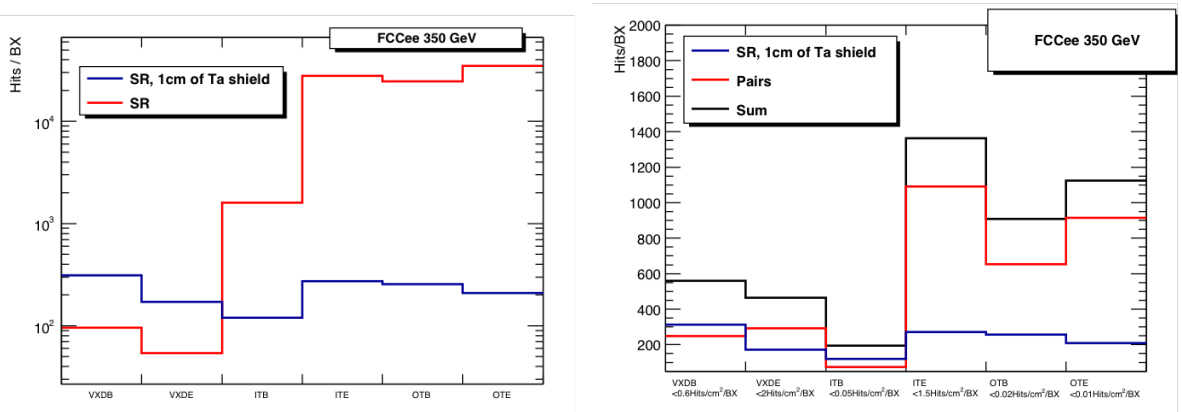

Figure 5: Left plot: effect of synchrotron radiation in all subdetectors with and without $1 \mathrm{~cm}$ Ta shielding; right plot: combined effect of synchrotron radiation and pair backgrounds.

\section{Conclusion and future steps}

We have described the machine detector interface studies with the interaction region optics, main parameters, the principle of the solenoid compensation scheme, and full simulation of the backgrounds studies with particular attention to synchrotron radiation. We have described the interaction region layout. We also showed in the layout the location of the luminosity monitor. It aims at an absolute precision in the measurement as high as $10^{-4}$ at the $\mathrm{Z}$, so that so a control of geometry to few $\mu \mathrm{m}$ is needed.

\section{Acknowledgments}

The author thanks all the FCC-ee MDI and optics teams, and in particular N. Bacchetta, E. Belli, A. Blondel, A. Bogomyagkov, H.Burkhardt, M. Dam, M. Gil Costa, A. Kolano, P. Janot, M. Koratzinos, E. Levichev, A. Novokhatski, K. Oide, E. Perez, S. Sinyatkin, M. Sullivan, G. Voutsinas, J. Wenninger, F. Zimmermann. 


\section{References}

[1] The FCC-ee study, http://cern.ch/fcc .

[2] A. Blondel, P. Janot, K. Oide, D. Shatilov, F. Zimmermann, FCC-ee parameter update, 6 October (2017).

[3] P. Raimondi, D. Shatilov, M. Zobov, arXiv:physics/0702033 (2007).

[4] K. Oide et al., Phys. Rev. Accel. Beams 19 (2016) no.11, 111005 Addendum: [Phys. Rev. Accel. Beams 20 (2017) no.4, 049901]

[5] K. Oide et al., doi:10.18429/JACoW-IPAC2017-TUOCB1

[6] G. von Holtey et al., Nucl. Instrum. Meth. A 403 (1998) 205.

[7] K. Ohmi, presented at the FCC WEEK 2016, Rome (April 2016).

[8] D. Shatilov, presented at the eeFact2016, Daresbury (October 2016).

[9] K. Ohmi, N. Kuroo, K. Oide, D. Zhou and F. Zimmermann, Phys. Rev. Lett. 119 (2017) no.13, 134801.

[10] M. K. Sullivan, presented at the FCC WEEK 2017, Berlin (2017).

[11] A. Novokhatski, presented at the FCC WEEK 2017, Berlin (2017).

[12] M. Boscolo and H. Burkhardt, IPAC-2015-TUPY004.

[13] M. Boscolo, H. Burkhardt and M. Sullivan, Phys. Rev. Accel. Beams 20 (2017) no.1, 011008.

[14] M. Koratzinos et al., doi:10.18429/JACoW-IPAC2016-THPOR023

[15] G. Voutsinas, N. Bacchetta, M. Boscolo, P. Janot, A. Kolano, E. Perez, M. Sullivan and N. Tehrani, doi:10.18429/JACoW-IPAC2017-WEPIK004

[16] CLICdp, http://clicdp.web.cern.ch 